\title{
Dynamics of the Halo of the Andromeda Galaxy M31
}

\author{
C. Halliday, D. Carter, Z. C. Jackson \\ Liverpool John Moores University, United Kingdom
}

T. J. Bridges, N. W. Evans, M. I. Wilkinson

AAO, Australia; Theoretical Physics, Oxford; IoA, Cambridge

\begin{abstract}
We present an investigation of the halo dynamics of M31 using planetary nebulae velocities. We have performed on-band [OIII] and off-band continuum imaging for a 3.6 square degree area centred on M31 and follow-up spectroscopy for over 600 planetary nebulae candidates. In the future the halo mass will be measured and the mass distribution and velocity anisotropy will be constrained as a function of radius.
\end{abstract}

\section{Introduction}

In cosmological N-body CDM simulations, galaxies form embedded within darkmatter halos. As resolution and predictive power on galaxy scalelengths of simulations increase, measurements of the halo density profiles for nearby galaxies provide important constraints of such models and their assumptions (e.g. cosmology). For spiral galaxies, strong evidence for the presence of dark-matter halos is provided by flat HI rotation curves. The decomposition of such data into a galaxy bulge, disk and dark-matter halo is highly degenerate - the use of tracer particles offer more powerful constraints.

Planetary nebulae $(\mathrm{PNe})$ are excellent tracers of the galaxy's potential particularly for large galactocentric radii where integrated stellar light measurements are difficult to obtain. The velocities of planetary nebulae have been used to investigate the dynamics of elliptical galaxies (see contributions by Arnaboldi and Freeman this conference). They are readily detectable due to their bright [OIII] emission lines using [OIII] imaging surveys and follow-up spectroscopy, or counter-dispersed imaging (Douglas, this conference). Modelling techniques to constrain galaxy halo mass and velocity anisotropy have been established that use satellite galaxy and globular cluster velocities (Evans \& Wilkinson 2000), or integrated light close to galaxy centre and tracer particle velocities at larger radii (e.g. Romanowsky \& Kochanek, 2000; Saglia et al., 2000).

\section{Dynamics of M31}

Measuring the mass density profile of the halo of M31 will considerably enhance the interpretation of ongoing microlensing experiments, e.g. POINT-AGAPE, WeCAPP and MEGA. For example, Kerins et al. (2001) present microlens- 
ing event predictions to constrain the galaxy MACHO mass fraction which are dependent on their assumed dynamical model for M31. High resolution cosmological N-body simulations for the Local Group are sensitive to the masses of both the Milky Way and M31.

\subsection{Previous work}

Kent (1989) presented new velocities for $30 \mathrm{HII}$ regions and performed an accurate comparison between HII and HI 21-cm data finding excellent agreement. Kent, Huchra \& Stauffer (1989) constrained the division of mass between the disk and halo using velocities for 149 globular clusters (GCs) and assuming velocity isotropy, finding the disk to contain between $\frac{1}{2}$ and all of the galaxy mass. Evans \& Wilkinson (2000) (EW00) measured the halo mass using velocities for satellite galaxies, PNe and GCs and found the mass to be less than their measurement for the Milky Way (Wilkinson \& Evans, 1999). Evans et al. (2000) revised their estimate of halo mass using tracer velocities for the dwarf spheroidal companions of M31 measured using the Keck HIRES/LRIS. Their measurement of $7.0_{-3.5}^{10.5} \times 10^{11} \mathrm{M}_{\odot}$ has far smaller uncertainty than for EW00.

\subsection{Imaging}

We have completed [OIII] and Stromgren Y continuum imaging for a $~ 3.6$ square degree field centred on M31 using the Wide Field Camera of the Isaac Newton Telescope on La Palma. In preparation for our most recent spectroscopic observations in October 2001 we detected over 1200 PNe candidates ${ }^{15}$ by blinking [OIII] and continuum images. Astrometry was performed using the Starlink tool GAIA. We plan to achieve automated detection of PNe candidates and astrometry for these data using $\mathrm{ARK}^{16}$ algorithms developed by T. Naylor. Candidates are selected based on the relative measurements of their [OIII] and Stromgren Y magnitudes. Astrometry will be bootstrapped using the positions of fiducial stars measured by N. Hambly for POSS-II images scanned using the SuperCOSMOS plate measuring machine. We estimate that this will enhance our current sample by an additional factor of $\sim 20 \%$. In the future we propose to extend our imaging survey from $20 \mathrm{kpc}$ to $30 \mathrm{kpc}$.

\subsection{Spectroscopy}

WHT WYFFOS spectra were obtained for 294 PNe from the sample detected by Ciardullo et al. (1989) (C89). Astrometry was completed by the careful translation of the epoch 1975 coordinates of C89 to J2000 positions. The spectral resolution was $1 \AA$ FWHM and examples of our spectra are presented in Fig. 1, while locations of candidates are shown in Fig. 2. In October 2001 WYFFOS spectroscopy for an additional $\sim 600 \mathrm{PNe}$ was obtained and data reduction of

${ }^{15}$ Our survey is sensitive to the detection of HII regions but since the investigation of dynamics requires halo mass potential tracers this is not considered to be a problem. Hereafter, we refer to $\mathrm{PNe}$ candidates as PNe.

${ }^{16} \mathrm{ARK}$ data reduction system is a collection of common user programs originally written by the Oxford High Energy Astrophysics Group 

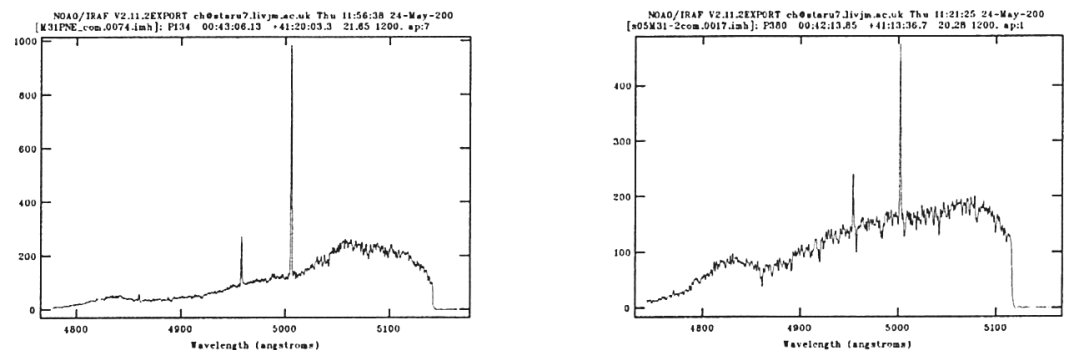

Figure 1. Examples of our WYFFOS spectra. Our wavelength range of $350 \AA$ is centred between the [OIII] $\lambda 4959$ and [OIII] $\lambda 5007$ emission lines.

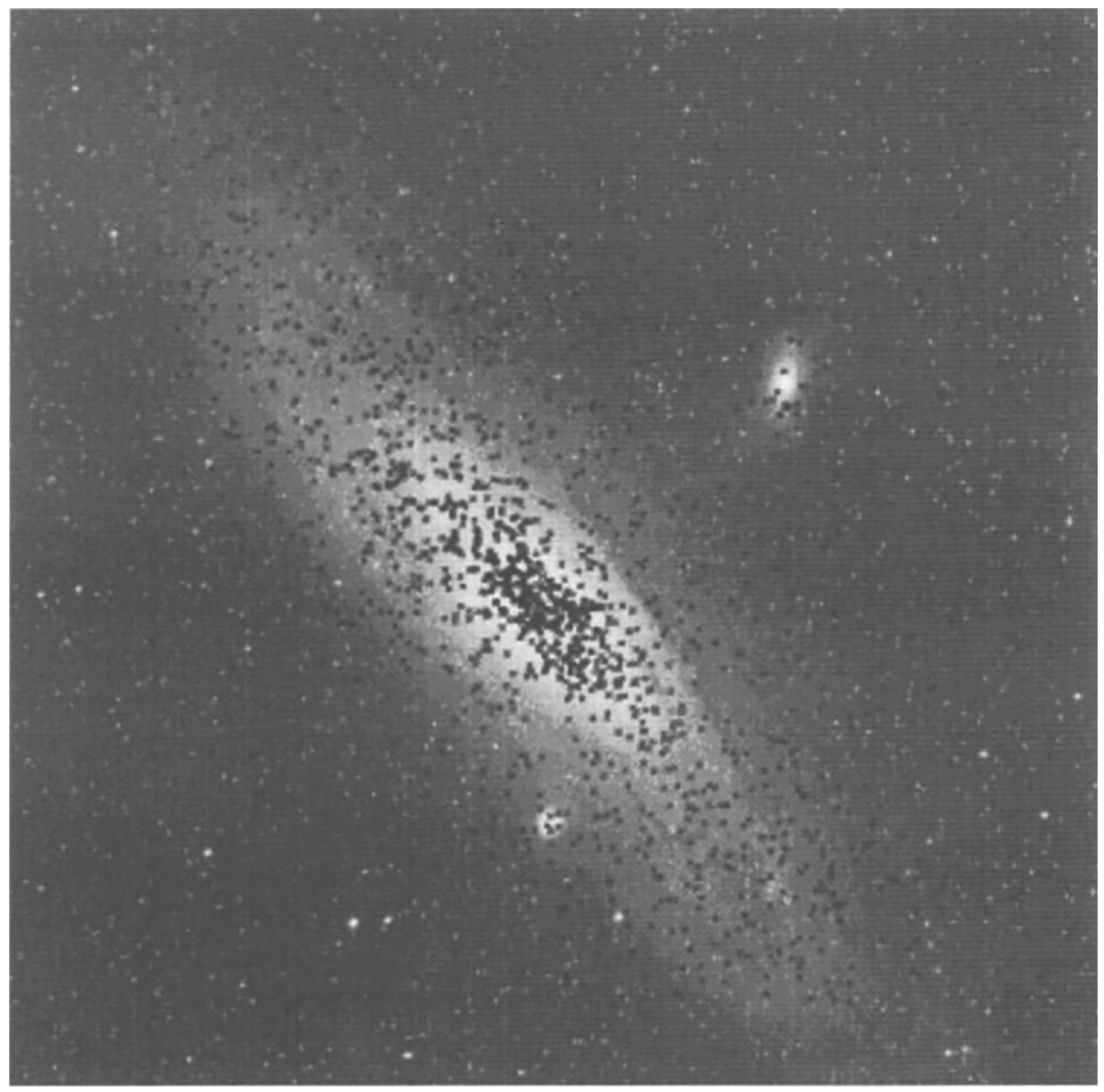

Figure 2. Positions of PNe observed adapting astrometry of C89 to J2000 (circles) and the 1284 PNe detected using our imaging (crosses) overplotted on image taken from the Digital Sky Survey. 
this data is in progress. We propose to obtain follow-up spectroscopy for an additional $\sim 600 \mathrm{PNe}$ detected using our imaging.

\subsection{Future Dynamical Modelling}

There is a large uncertainty in the mass of M31. Partly this is because of the paucity of tracers of the potential at large radii. Population II stars are much too faint and samples of globular clusters have been too small until very recently. Partly this is because of the well-known degeneracy between mass and anisotropy in stellar kinematics. For example, an anisotropic system with orbits that become more and more circular with increasing radius typically has a velocity dispersion that rises with radius, mimicking a massive dark halo.

There have been a number of techniques developed in recent years to analyse samples of discrete radial velocities. The simplest involve variants of the virial theorem. These are typically weighted sums of squares of velocities and projected positions and involve an assumption as to the average velocity anisotropy of the tracer population. They are simple, but they come with the price of strong assumptions. A more powerful approach is to build dynamical models of tracer populations in steady-state trial potentials. This gives us the probability distribution of observing a star of a given component - for example a PNe or GC - at any location. Such probabilities may then need convolution with the selection functions of the survey. The final probability distribution is evaluated at the location of each star to give the likelihood of the data assuming the model. This likelihood is then maximised by adjusting a suitably parametrised form of the potential, as well as functions that characterise each component (such as the PNe or GCs).

The new PNe velocities, together with the database of M31 GC velocities assembled by K. Perrett and collaborators and the more distant dwarf spheroidal satellite galaxies, will provide the largest sample of halo tracers yet assembled for any spiral galaxy. We are analysing this new database with the models of EW00 to determine the halo mass profile of M31. This will provide the best etsimates to date of the mass of M31's halo, together with the the mean rotation and velocity anisotropy for the separate PNe and GC populations.

\section{References}

Ciardullo, R., Jacoby, G. H., Ford, H. C., \& Neill, J. D. 1989, ApJ, 339, 53

Evans, N. W., \& Wilkinson, M. 2000, MNRAS, 316, 929

Evans, N. W., Wilkinson, M., Guhathakurta, P., Grebel, E. K. \& Vogt, S. S. 2000, ApJ, 540 , L9

Kent, S. M. 1989, PASP, 101, 489

Kent, S. M., Huchra, J. P., \& Stauffer, J. 1989, AJ, 98, 2080

Kerins, E., Carr, B. J., Evans, N. W., Hewett, P., Lastennet, E., Le Du, Y., Melchior, A.-L., Smartt, S. J., \& Valls-Gabaud, D. 2001, MNRAS, 323, 13

Romanowsky, A. J., \& Kochanek, C. S. 2000, ApJ, 553, 722

Saglia, R. P., Kronawitter, A., Gerhard, O., \& Bender, R. 2000, AJ, 119, 153

Wilkinson, M., Evans, N. W. 1999, MNRAS, 310, 645 